\title{
Somatotopy and Attentional Modulation of the Human Parietal and Opercular Regions
}

\author{
Jeremy P. Young, ${ }^{1}$ Priyantha Herath, ${ }^{2}$ S. Eickhoff, ${ }^{3,4}$ J. Choi, ${ }^{3,4}$ C. Grefkes, ${ }^{3,4}$ K. Zilles, ${ }^{3,4}$ and Per E. Roland ${ }^{1}$ \\ ${ }^{1}$ Division of Brain Research, Department of Neuroscience, Karolinska Institute, Stockholm Sweden S-171 77, ${ }^{2}$ Department of Neurology, University of \\ Pittsburgh School of Medicine, Pittsburgh, PA 15260, ${ }^{3} \mathrm{C}$. and O. Vogt Brain Institute, University of Dusseldorf, Germany 40001, and ${ }^{4}$ Institute of Medicine, \\ Research Center Julich, Germany 52425
}

The somatotopical organization of the postcentral gyrus is well known, but less is known about the somatotopical organization of area 2 , the somatosensory association areas in the postparietal cortex, and the parietal operculum. The extent to which these areas are modulated by attention is also poorly understood. For these reasons, we measured the BOLD signal when rectangular parallelepipeds of varying shape were presented to the immobile right hand or right foot of 10 subjects either discriminating these or just being stimulated. Activation areas in each subject were mapped against cytoarchitectural probability maps of area 2, IP1, and IP2 along the intraparietal sulcus and the parietal opercular areas 0P1-0P4.

In area 2, the somatotopical representation of the hand and foot were distinctly separate, whereas there was considerable overlap in IP1 and no clear evidence of separate representations in OP1, OP4, and IP2. The overlap of hand and foot representations increased in the following order: area 3a, 3b, 1, 2, IP1, OP4, IP2, and OP1. There were significant foot representations but no hand representations in right (ipsilateral) areas $3 \mathrm{a}, 3 \mathrm{~b}$, and 1 . Shape discrimination using the foot as opposed to stimulation enhanced the signal in $0 \mathrm{P} 4$ bilaterally, whereas discrimination with the hand enhanced the signal bilaterally in area 2, IP1, and IP2. These results indicate that somatosensory areas in humans are arranged from strong somatotopy into no somatotopy in the following order: 3a, 3b, 1, 2, IP1, 0P4, IP2, and 0P1. Higher order areas such as IP1, IP2, and OP4 showed task-related attentional enhancement.

Key words: somatotopy; attention; somatosensory; parietal; operculum; cortex

\section{Introduction}

The human postcentral gyrus is associated with somatic sensation, and an orderly representation of the skin surface (somatotopy) has been demonstrated with various methods (Head and Holmes, 1911; Foerster, 1936; Penfield and Boldrey, 1937; Corkin et al., 1970). Using an observer-independent method in a small population, a subdivision of the human postcentral gyrus has recently revealed probability maps for areas $3 \mathrm{a}, 3 \mathrm{~b}, 1$, and 2 (Geyer et al., 1999; Grefkes et al., 2001). It is not known whether each of these areas contain a full representation of the skin surface.

There are additional somatosensory areas in the parietal operculum and perhaps the retroinsular cortex, but their number and location are still unresolved (Penfield and Rasmussen, 1950; Hari et al., 1983; Roland and Mortensen, 1987; Seitz and Roland, 1992; Burton et al., 1993, 1997; Lin et al., 1996; Hodge et al., 1998;

Received June 26, 2003; revised April 29, 2004; accepted April 29, 2004.

This project was supported by European Union Grant DCG-CT-1999 - 00677 Neurogenerator and by a grant from the Swedish Research Council. The cytoarchitectonic part of this work was supported by Deutsche Forschungsgemeinschaft grants (K.Z.), and Human Brain Project/Neuroinformatics was funded by the National Institute of Biomedical Imaging and Bioengineering, the National Institute of Neurological Disorders and Stroke, and the National Institute of Mental Health (K.Z.).

Correspondence should be addressed to Jeremy Young, Division of Human Brain Research, Department of Neuroscience, Retzius väg 8, A3:3, Karolinska Institute, Stockholm, Sweden S-171 77. E-mail: Jeremy.Young@neuro.ki.SE.

D0I:10.1523/JNEUROSCI.4030-03.2004

Copyright $\odot 2004$ Society for Neuroscience $\quad 0270-6474 / 04 / 245391-09 \$ 15.00 / 0$
Maldjian et al., 1999; Polonara et al., 1999; Disbrow et al., 2000; Francis et al., 2000; Corbetta et al., 2002; Hagen and Pardo, 2002). Similarly, areas with predominantly somatosensory functions have been described lining the anterior part of the intraparietal sulcus and in the superior parietal lobule (Penfield and Rasmussen, 1952; Roland, 1984; Seitz et al., 1991; O’Sullivan et al., 1994; Burton et al., 1996; Matsumura et al., 1996; Roland et al., 1998). For these areas to qualify as somatosensory areas in humans, a prerequisite would be a full representation of the skin surface (Kaas et al., 1979). In addition, these tentative somatosensory areas may be arranged on the basis of progressive larger receptive fields. A consequence of larger receptive fields could be a decreasing somatotopical organization. One may even expect a transition from somatotopical organization to a nonsomatotopical organization in analogy with higher order visual areas in the inferotemporal cortex of the monkey (Wang et al., 1996). An increase in focal attention can also enhance activity in somatosensory areas in the postcentral gyrus (Roland, 1981), and it is claimed that the effects of attention in behavioral tasks are stronger for higher order sensory areas.

Choi et al. (2002) and Eickhoff et al. (2002) have recently published a cytoarchitectural division of areas lining the anterior and middle part of the human intraparietal sulcus, area IP1 and IP2, the parietal operculum, areas OP1, OP2, OP3, and OP4. Based on this, we examined the hypotheses that (1) each of the presently known and newly delimited cytoarchitectural areas in 
Table 1. Dimensions of the rectangular parallelepipeds used for stimulating the hand and foot

\begin{tabular}{lllll}
\hline Hand & & & Foot \\
\cline { 5 - 5 } Object number & Dimensions $(\mathrm{mm})$ & & Object number & Dimensions $(\mathrm{mm})$ \\
\hline Cubic & $22.57 \times 22.54$ & & Cubic & $22.57 \times 22.54$ \\
2 & $23.01 \times 22.37$ & 5 & $24.47 \times 21.64$ \\
4 & $24.01 \times 21.89$ & & 7 & $25.49 \times 21.23$ \\
6 & $24.98 \times 21.44$ & & 10 & $26.48 \times 20.81$ \\
7 & $25.49 \times 21.23$ & & 11 & $31.49 \times 19.11$ \\
8 & $26.48 \times 20.81$ & & 14 & $39.52 \times 17.06$
\end{tabular}

Two sets of six objects for stimulation of the hand and another set for stimulation of the foot were chosen for comparison with the cubic object (object number 1). The object number and dimensions (in millimeters) appear in the table. Dimensions of the rectangular parallelepipeds were used to stimulate the hand and foot.

the postcentral gyrus, anterior parts of the parietal lobules, and parietal operculum contain somatosensory representations of the hand and foot; (2) some of these areas (2, IP1, IP2, OP1-OP4) will have less or no somatotopical organization, and hence be classified as higher order somatosensory areas; and (3) areas, according to hypothesis 2, classified as higher will show attentiondependent signal enhancement in task-relevant conditions. To examine these hypotheses, we compared passive stimulation with rectangular parallelepipeda to either the right hand or foot with a condition of active discrimination. These procedures have been shown previously to activate potential somatosensory areas in the parietal cortex (Roland and Larsen, 1976; Bodegard et al., 2001).

\section{Materials and Methods}

The Karolinska Hospital Ethics Committee approved this study. Ten healthy subjects, six males and four females, 21-32 years of age (mean age, 26.8 years) gave informed written consent in accordance with the Helsinki Declaration. All subjects were strongly right handed according to the Edinburgh Inventory (Oldfield, 1971), which was given in English or a version translated into Swedish, depending on the subjects' native language. None of the subjects had a previous history of neurological disease and had normal magnetic resonance (MR) brain images. Psychophysical measurements of the behavioral tasks were performed on all subjects before MR scanning.

Stimuli. Rectangular parallelepipeds were used as stimuli. Fifteen rectangular parallelepipeds varied in shape from cubic $(22.57 \times 22.54 \mathrm{~mm})$ to more oblong $(44.53 \times 16.06 \mathrm{~mm}$ ) (Roland, 1975; Roland and Mortensen, 1987). The shapes were custom made from polyvinyl chloride material compatible with the MR environment. All parallelepipeds were of the same weight, microgeometric surface properties, and thermal properties. Therefore, the objects differed only in shape. Subjects were only shown the objects on completion of the experiment.

Before functional MR scanning, psychophysics was performed on all subjects to determine the discrimination thresholds (Roland and Mortensen, 1987) of the rectangular parallelepipeds. From these psychophysical results, six parallelepiped objects were selected for comparison with the most cubic object (Table 1) such that subjects could determine the cubic object with a probability of detection between 0.7 and 0.8 . A set of six objects was used on the hand, and another set of six objects was used for the foot (Fig. 1).

Stimulus location. Stimulation was performed on the right hand or the right foot. To determine the area of stimulation, a parallepiped was dipped in stamp ink and the shape was passively rotated on the right hand and foot. On the right hand, the area of stimulation included the distal palmar surface adjacent to and including the proximal phalanges of digits 2,3 , and 4 . Depending on the size of the object, the area of stimulation of the palmar surface ranged from 2036 to $2797 \mathrm{~mm}^{2}$ for all subjects. On the right foot, the area of stimulation included the proximal and distal phalanges of digits 1,2, and 3 and adjacent parts of the planta. For all subjects, the area of stimulation of the planta ranged from 2036 to $3584 \mathrm{~mm}^{2}$ for the most cubic to the most oblong object, respectively.

Presentation of parallelepiped objects. To minimize any bias in the ob-

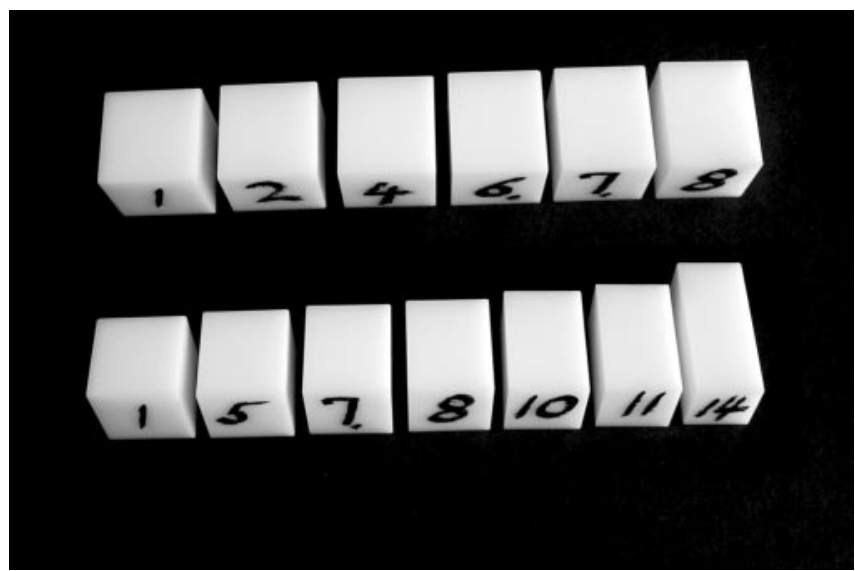

Figure 1. The set of six parallelepiped objects used for passive stimulation of the palmar surface on the hand (top set) and the planta surface on the foot (bottom set). For the discrimination tasks, all objects were compared with the most cubic object, object number 1 .

ject stimulations, the following steps were taken. All stimulations were performed by the same author, and identical stimulations were applied on two different occasions. For psychophysical testing and functional MR imaging (fMRI) scanning so that both the author and subject were familiar with all tasks, all subjects had to complete psychophysical testing with a probability of being able to discriminate correctly the cube with an accuracy of at least 0.7 on both the hand and foot, and psychophysical performance was measured during the scanning sessions. Timing was kept by the MRI clock displaying the remaining scan time.

All object presentations were standardized and performed on the subject's right hand or foot. Each parallelepiped was passively rotated over four surfaces in a systematic order. The object was always in contact with the skin while being rotated. The rotation consisted of five systematic moves. For the hand, the shortest side was first placed on the distal palmar surface and sequentially rotated onto the long side on the proximal phalanges 2 and 3 . Then, the object was rotated onto the next long side on phalanges 3 and 4 followed by a rotation back to the same short side on the distal palmar surface adjacent to digits 3 and 4 and then rotated onto the long side on the distal palmar surface adjacent to digits 2 and 3 . The object was then removed. The stimulus duration was 3.5 sec. An identical procedure was performed on the right foot area of stimulation.

A two-alternative forced choice (2-AFC) paradigm was used to determine the discrimination limits of distinguishing rectangular parallaepipeds from the cube. In each trial, a parallelepiped was compared with the cube. Each trial lasted $10 \mathrm{sec}$. In the first $3.5 \mathrm{sec}$, either the parallelepiped or the cube was presented; in the next $3.5 \mathrm{sec}$, the object for comparison was presented, which was the cube in the case where the parallelepiped was presented in the first interval, or the parallepiped in case where the cube was presented in the first interval. Thus, the cube was the standard appearing in each 2-AFC trial. Immediately after the second object presentation, a verbal response of "one" or "two" was required, depending on which object, the first or the second, respectively, the subject thought was cubic.

Conditions. There were five experimental conditions: (1) discriminating objects on the hand (HD) and (2) on the foot (FD), and (3) object stimulation on the hand (HS) and (4) on the foot (FS), and (5) a rest condition. During all conditions, subjects closed their eyes and wore a blindfold and headphones, through which they were informed at all times about the condition they were about to perform. The order of which the tasks were presented to the subjects was randomized for each scanning session.

Shape discrimination on the hand. For this condition, subjects were instructed that two objects would be passively presented on their right hand, one after the other. They could decide which of the two objects, the first or the second, was the cube. When the second object presentation was complete, they could verbally respond one or two, depending on 
which of the two objects, the first or the second, respectively, was more cubic.

Shape discrimination on the foot. This condition is the same as the HD condition, except that the instructions and stimulation with the object were adopted for the right foot.

Same shape on the hand. The subjects were instructed that an object would be passively presented twice to the right hand. Because the object was identical both times, no discrimination was needed. On completion of the second presentation, subjects verbally responded with two.

Same shape on the foot. This condition is similar to the HS condition; however, the difference was that the right foot was used for stimulation. The subjects were instructed that an object would be passively presented twice to the right foot. Because the object was identical both times, no discrimination was needed. On completion of the second presentation, the subjects verbally responded with two.

Rest. During the rest condition, subjects were instructed to close their eyes, relax, and not to think of anything in particular. Headphones and a blindfold were worn over the ears and eyes, respectively. On completion of a scan, subjects were questioned as to whether they heard or felt anything during the scan that disturbed them.

Scanning procedure. Subjects lay comfortably in the supine position in the MR camera. A thermoplastic bite piece was used to stabilize the subject's head for the duration of the experiment. The subject's feet and hands were exposed and rested in a comfortable position so that the palmar surface of the hand faced upwards and the sole of the foot was vertical. Subjects were blindfolded and wore noise-attenuating headphones. Instructions were given to the subject via the headphones before each condition.

MR data were acquired on a clinical GE Signa $1.5 \mathrm{~T}$ scanner (Signa Horizon Echospeed; General Electric, Milwaukee, WI) fitted with a head coil. An anatomical, high-resolution, three-dimensional, gradient-echo, T1-weighted volume of the whole brain was acquired with a spoiled gradient-recalled acquisition in a steady state sequence [echo time (TE), $4.2 \mathrm{msec}$; repetition time (TR), $13 \mathrm{msec}$; field of view (FOV), $240 \mathrm{~mm}$; flip angle, 40 ; voxel size, $0.96 \times 0.96 \times 2.0 \mathrm{~mm}^{3}$ ]. Functional data were acquired with T2-weighted blood-oxygenated level-dependent (BOLD) signals measured with an echo-planar image sequence (TE, $60 \mathrm{msec}$; TR, $5000 \mathrm{msec}$; flip angle, 90 ; $64 \times 64$ matrix; FOV, $240 \mathrm{~mm}$; voxel size, $3.4 \times$ $3.4 \times 5 \mathrm{~mm}^{3}$ ). A functional volume comprised 21 contiguous axial slices. The first $20 \mathrm{sec}$ of data were discarded. A functional scanning session lasted $270 \mathrm{sec}$ ( 54 volumes). A block design paradigm was used so that one scanning session consisted of $9 \times 30 \mathrm{sec}$ blocks. Each $10 \mathrm{sec}$ trial was repeated three times within a block. Odd blocks were rest conditions. Each of the four even blocks was one of the HD, FD, HS, or FS conditions randomized across scanning sessions. Scan sessions were repeated 16 times for each subject. Of the 864 volumes acquired in total, 480 volumes were of the rest state and 96 volumes were acquired for each of the HD, $\mathrm{FD}, \mathrm{HS}$, and FS conditions.

Image analysis. Analysis of the reconstructed functional imaging data was performed with statistical parametric mapping 99 (SPM99) (Wellcome Department of Cognitive Neurology, UK; http://www.fil.ion.ucl.ac.uk/spm). Data from each subject were treated separately. First, functional data for each subject was corrected for movement with a least squares fit and six-parameter rigid body spatial transformation. Functional and high-resolution anatomical images were coregistered. Anatomical images and subsequently the functional images were aligned to the space of Talairach and Tournoux (1988) with the standard brain (Roland et al., 2001) as a template. Alignment involved the estimation of the optimum 12-parameter affine registration followed by the estimation of nonlinear deformations and three-dimensional discrete cosine transform basis functions. The data were smoothed with an isotropic Gaussian filter with a full width at half maximum (FWHM) of $6 \mathrm{~mm}$. Time series data were temporally smoothed with a Gaussian filter with FWHM of 4 sec.

Individual analysis. Condition-specific effects for each subject were estimated with a fixed-effect general linear model convolved with a $4 \mathrm{sec}$ delayed boxcar waveform as implemented in SPM99. Realignment parameters were included as covariates in the linear model.

Statistical inferences were made at the cluster level such that the om- nibus probability of detecting a cluster $>100$ voxels is $<0.05$. From the cytoarchitectural probability map, a volume of interest covering area 6 , $4 \mathrm{a}, 4 \mathrm{p}, 3 \mathrm{a}, 3 \mathrm{~b}, 1,2$, OP areas, IP areas, and the parietal lobe was used to correct the $p$ values. Boolean intersections were made between brain activation results from multiple contrasts.

Group analysis. A random-effects analysis (Friston et al., 1999) was performed to reveal the group activations for somatotopy and the effect of task-related attention. To examine somatotopy of the group, a random-effect analysis of the contrasts $[(\mathrm{HD}+\mathrm{HS})-2 R]$ and $[(\mathrm{FD}+$ FS) $-2 R]$ was made (Table 2). To examine the effect of task-related attention from the group, a random-effect analysis of the contrasts $[(\mathrm{HD}-\mathrm{HS})$ and $(\mathrm{FD}-\mathrm{FS})]$ was used.

Cytoarchitectonic mapping. Cytoarchitecturally delineated areas 6, 4a, $4 \mathrm{p}, 3 \mathrm{a}, 3 \mathrm{~b}, 1,2$, IP, and OP areas were used for localizing the activations. Delineation of all cytoarchitectonic areas used in this study, including areas 4a and 4p (Geyer et al., 1996), 3a, 3b, 1, (Geyer et al., 1999), and 2 (Grefkes et al., 2001) that have been published previously, all use the same procedures described below. Briefly, histological serial sections (20 $\mu \mathrm{m}$ thick) of 10 postmortem adult human brains were silver stained for cell bodies. The histological sections were matched with a previously acquired MR image of the same brain before fixation and sectioning to account for histological procedural artifacts. A quantitative observerindependent method based on the gray level index was used to measure cortical laminar densities of neurons and to determine borders between cytoarchitectonic areas for each brain (Schleicher et al., 1999). Each delineated cytoarchitectural area was reconstructed as a three-dimensional digital image aligned to the standard brain (Roland et al., 2001) and filtered with an isotropic Gaussian filter with an FWHM of $6 \mathrm{~mm}$. A population map (Roland and Zilles, 1998) was generated by superimposing corresponding cytoarchitectural areas of each postmortem brain in standard anatomical three-dimensional space of the human brain atlas (Roland et al., 2001). From the population map, each cytoarchitectural area was defined by voxels having a higher probability of belonging to this area than to any other area. This produced the $6 \mathrm{~mm}$ filtered cytoarchitectural probability map (Bodegard et al., 2001).

The Boolean intersection of all foot versus rest and all hand versus rest was made: $[(\mathrm{FD}+\mathrm{FS})-2 R] \cap[(\mathrm{HD}+\mathrm{HS})-2 R]$ and $[(\mathrm{FD}+\mathrm{FS})-$ $2 R] \cap[(\mathrm{HD}+\mathrm{HS})-2 R)]$. To localize the activations, the intersected images were superimposed on the $6 \mathrm{~mm}$ filtered cytoarchitectural probability map. Overlap of all hand intersections with all foot $(\mathrm{H} \cap \mathrm{F})$ and all hand unions with all foot $(H \cup F)$ of the activation images with each area in the cytoarchitectural probability map was calculated (see Fig. 6). The $\mathrm{H} \cap \mathrm{F} / \mathrm{H} \cup \mathrm{F}$ ratio represents the commonality between hand and foot activations for each subject.

\section{Results}

\section{Psychophysics}

During the scanning, the subjects were on average able to discriminate correctly the cube on the right foot (FD) with 0.80 probability and on the right-hand (HD) with 0.81 probability. A psychophysical curve averaged over 10 subjects is shown in Figure 2 .

\section{Imaging representations of hand and foot in individual subjects}

A probability map of cytoarchitectural areas 3a, 3b, 1, 2, IP1, IP2, and OP1-OP4 of the cerebral cortex is depicted in Figure 3. The probability map gives the best estimate of localizing a cytoarchitectural area. We will focus on hand and foot representations within the somatosensory and putative somatosensory areas: $3 \mathrm{a}$, 3b, 1, 2, IP1, IP2, and OP1-OP4. The location of areas 3a, 3b, and 1 (Geyer et al., 1999) and area 2 (Grefkes et al., 2001) has been reported previously. More caudally and abutting area 2 is area IP1, lining the rostral part of the intraparietal sulcus. Caudally and somewhat medially to IP1 and abutting IP1 is area IP2 (Choi et al., 2002) (Fig. 3a,c). The four opercular areas are situated in the parietal operculum in the cortex lining the foot of the lateral 
Table 2. Group results from a random-effect analysis

\begin{tabular}{|c|c|c|c|c|c|c|}
\hline \multirow[b]{2}{*}{ Contrast } & \multirow[b]{2}{*}{ Anatomical location of peak activity } & \multirow{2}{*}{$\begin{array}{l}\text { Cluster size }\left(\mathrm{mm}^{3}\right) \text { at } \\
\text { omnibus } p<0.05\end{array}$} & \multicolumn{3}{|c|}{$\begin{array}{l}\text { Talairach coordinate of the peak within the } \\
\text { cluster }\end{array}$} & \multirow[b]{2}{*}{ Voxel level Z-score } \\
\hline & & & $x$ & $y$ & $Z$ & \\
\hline \multirow[t]{11}{*}{ All hand versus rest } & Left 3a, 3b, 2, IP1, IP2, 0P1 & 1791 & -34 & -36 & 40 & 5.34 \\
\hline & Right thalamus & 336 & 14 & -10 & -4 & 5.08 \\
\hline & Left putamen & 90 & -24 & -10 & 0 & 4.73 \\
\hline & Right IP1, IP2 & 275 & 38 & -50 & 38 & 4.65 \\
\hline & Left OP4 & 128 & -42 & -20 & 18 & 4.34 \\
\hline & Right 0P1, IP1 & 148 & 52 & -18 & 24 & 4.27 \\
\hline & Right posterior to OP4 & 60 & 62 & -34 & 14 & 4.19 \\
\hline & Left 6 & 98 & -8 & -14 & 48 & 4.11 \\
\hline & Right 44 & 27 & 50 & 6 & 26 & 4.09 \\
\hline & Right cerebellum & 25 & 10 & -58 & -12 & 3.90 \\
\hline & Right putamen & 32 & 24 & 4 & -4 & 3.78 \\
\hline \multirow[t]{13}{*}{ All foot versus rest } & Right 44, 0P1, 0P4 & 590 & 48 & 2 & 24 & 5.60 \\
\hline & Left OP1, OP2, OP4 & 1140 & -48 & -18 & 16 & 5.12 \\
\hline & Right IP2 & 178 & 28 & -50 & 32 & 4.70 \\
\hline & Left 6 & 236 & -14 & -18 & 44 & 4.64 \\
\hline & Right IP1, OP1 & 186 & 50 & -42 & 46 & 4.60 \\
\hline & Left 4a & 40 & -2 & -36 & 54 & 4.58 \\
\hline & Right 6 & 136 & 32 & -10 & 32 & 4.45 \\
\hline & Right thalamus & 66 & 6 & -26 & 0 & 4.43 \\
\hline & Right inferior frontal gyrus & 48 & 30 & 26 & 2 & 4.40 \\
\hline & Left 3b, 1 & 168 & -20 & -54 & 48 & 4.31 \\
\hline & Right 44 & 34 & 28 & 14 & 6 & 4.24 \\
\hline & Left 2, IP1, IP2 & 57 & -32 & -42 & 34 & 4.09 \\
\hline & Right 6 & 27 & 28 & -8 & 48 & 3.86 \\
\hline
\end{tabular}

The anatomical location, significant cluster size, Talairach coordinate, and peak voxel Z-score are shown for the contrasts of all foot versus rest and all hand versus rest.

\section{Psychophysical curve for object discrimination}

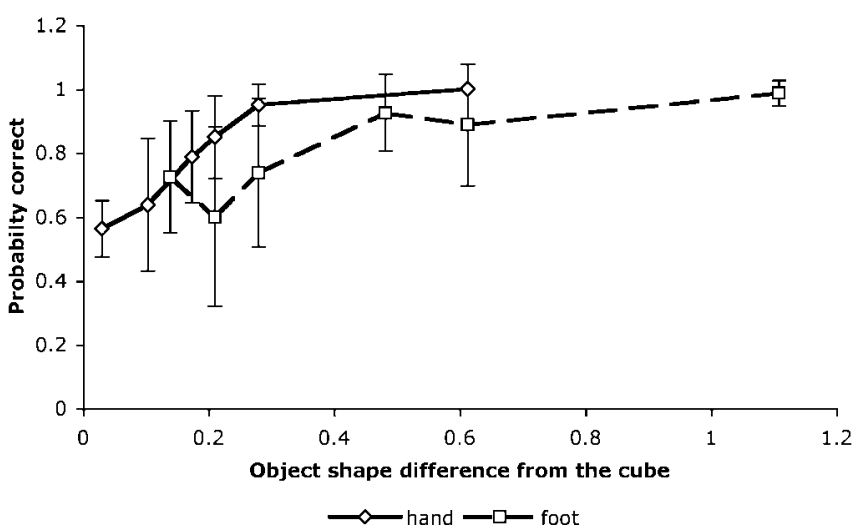

Figure 2. Psychophysical curve of the HD and FD tasks. The probability of choosing correctly the cubic object appears on the $y$-axis and the difference in object shape appears on the $x$-axis (calculated by

$$
\sqrt{\frac{4\left(\mathrm{a}_{1}-\mathrm{a}_{2}\right)^{2}+8\left(\mathrm{~b}_{1}-\mathrm{b}_{2}\right)^{2}}{8 a_{1}+16 \mathrm{~b}_{1}+16 \mathrm{~b}_{2}+8 \mathrm{a}_{2}}}
$$

where $a_{1}$ and $b_{1}$ are the short and long sides of the cubic object, and $a_{2}$ and $b_{2}$ are the short and long sides of the object for comparison). Both hand and foot psychophysics are represented in the figure.

sulcus (Eickhoff et al., 2002). OP2 and OP3 are localized most medial in the parietal operculum, with OP3 rostrally and OP2 caudally. OP4 is localized laterally in the operculum, lateral to OP3 and extending somewhat into the lateral surface of the supramarginal gyrus. OP1 is located caudally to OP4 also laterally in the parietal operculum and extending into the supramarginal gyrus (Fig. 3b).

\section{Individual analysis}

Using these cytoarchitectural areas as the best estimate of localization of somatosensory areas, we tested the hypothesis that each of these areas contains a representation of the hand and foot. For this purpose, the contrast of all foot versus rest $[(\mathrm{FD}+\mathrm{FS})-2 R]$ was made to reveal foot representations in each subject. Similarly, the contrast of all hand versus rest $[(\mathrm{HD}+\mathrm{HS})-2 R]$ was made to reveal the hand representations in each subject. A subject was considered to have a hand or a foot representation if the respective contrast revealed a statistically significant cluster size (at omnibus $p<0.05$ ). A summary of the 10 subjects is given below, and results from two complete individual cases are presented to illustrate significant clusters (Fig. 4). Cluster locations, sizes, and significance are also reported from a random-effects analysis of all subjects (Tables 3 ).

\section{Foot}

As the right planta and toes were stimulated, one would expect to find representations of the foot in the left hemisphere somatosensory areas. Indeed, there were foot representations in 9 of 10 subjects in the cortex of areas $3 \mathrm{~b}$ and 1 on the mesial surface of the left hemisphere. (Fig. 4, $a$ and $b$, illustrates these activations for subjects 1 and 2, respectively). For area 2, four subjects had a significant activity representing the foot located in the top part of the left area 2, above $\mathrm{z}=50$ (Fig. $4 a$ ). Because area 2 does not extend into the mesial surface, these foot activations thus were located on the lateral surface of the hemisphere. Eight subjects had foot representations in the left IP1 and nine subjects had representations in the left IP2 (Fig. $4 a, b$ ). Eight of the 10 subjects had a representation of the foot in the left OP1 and OP4 (Fig. $4 a, b)$. There were sparse representations of the foot in the parietal opercular areas OP2 and OP3 on the contralateral side. The right (ipsilateral) area OP3 primarily contained a representation of the foot.

As expected, the hand was represented in area $3 \mathrm{~b}$ and 1 clus- 


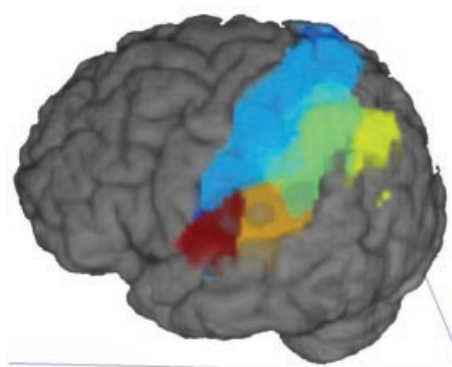

A
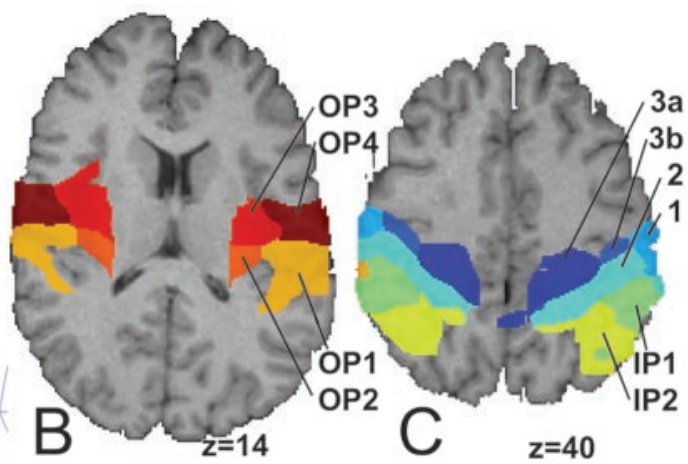

Figure 3. The locations of areas $3 \mathrm{a}, 3 \mathrm{~b}, 1,2, \mathrm{IP1}, \mathrm{IP} 2, \mathrm{OP} 1,0 \mathrm{P} 2, \mathrm{OP} 3$, and $0 \mathrm{P} 4$. The cytoarchitectural probability map of these areas was filtered with a $6 \mathrm{~mm}$ FWHM and overlaid on the standard brain. Areas 1, IP1, IP2, OP1, and OP4 can been seen in the surface render $(A)$; in addition, OP1, OP2, OP3, and OP4 can be seen in the axial slice $B$ and areas $3 \mathrm{a}, 3 \mathrm{~b}, 1,2, \mathrm{PP} 1$, and IP2 in axial slice $C$.
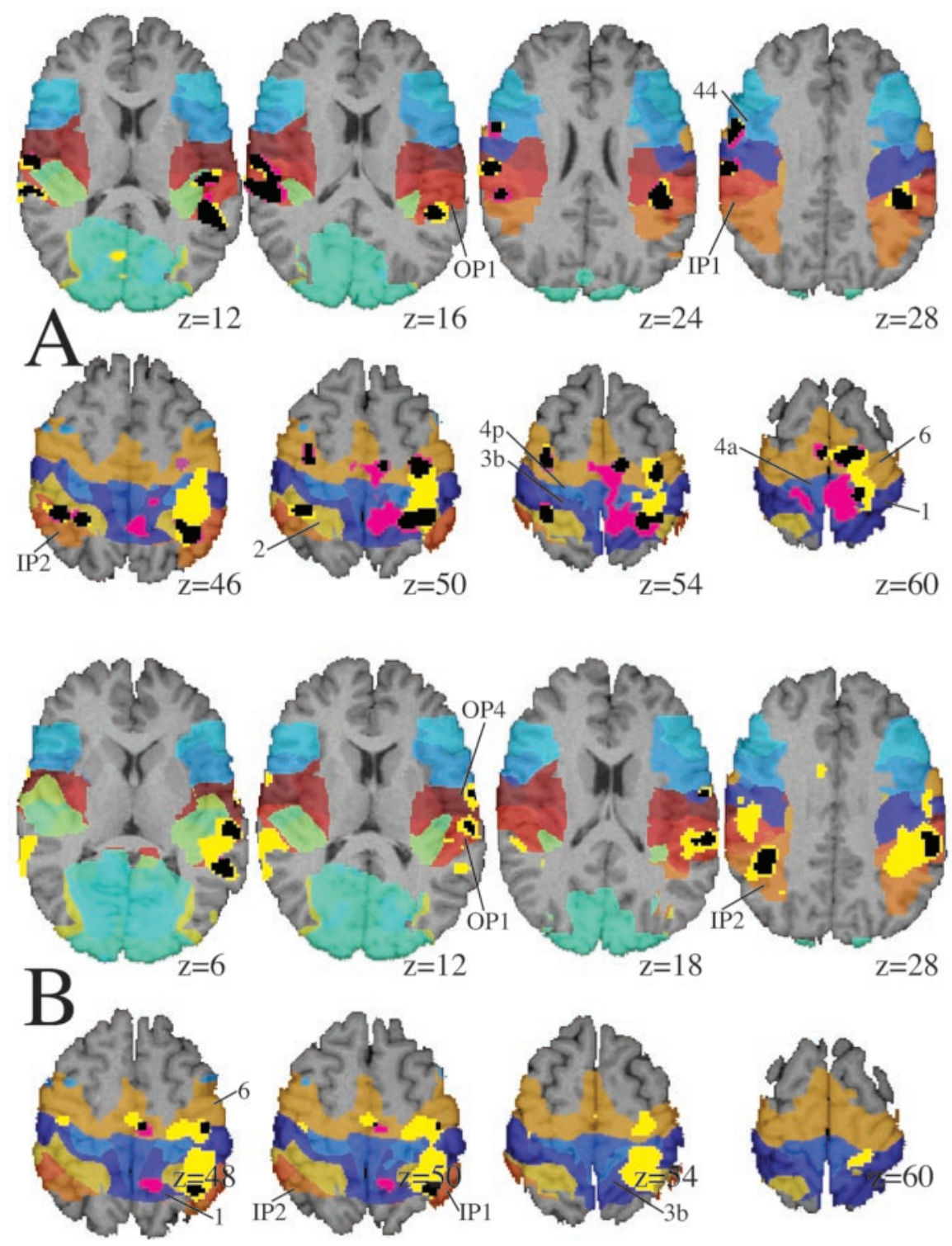

Figure 4. $\quad A, B$, Hand and foot representations in the cortex for individual subjects $1(A)$ and $2(B)$. Significant clusters are shown for contrasts of all hand versus rest (yellow) and all foot versus rest (purple). The overlap between hand and foot clusters appears as a solid black color. The activations appear as solid colors and are overlaid on the cytoarchitectural probability map (translucent color coded) and standard brain (gray scale). Images are in Talariach space, and the level of the axial slice is given by the $z$-coordinate. tered between Talairach coordinates $z=$ 36 and 60 . In addition, the hand representation of area 2 was in approximately the same position mediolaterally (Fig. $4 a, b$ ). The majority of the 10 subjects had statistically significant representations of the hand in areas IP2, OP1, and OP4 in the left hemisphere (Fig. 4a,b). There were many ipsilateral activations in these subjects. For the foot stimulation, these appeared even in ipsilateral areas 3b, 1, and 2 (Fig. 4d,e,g). As one proceeds further caudally, almost all subjects had significant clusters in ipsilateral areas IP1, IP2, OP1, and OP4 (Fig. 4). In these areas, there was no obvious segregation in the location of the clusters for the foot and the hand, either contralaterally or ipsilaterally. This is illustrated by the two individual subject results (Fig. $4 a, b)$.

\section{Somatotopy}

From the previous analysis of all foot versus rest and all hand versus rest, one can see that the hand and foot representations are somewhat segregated near the superolateral border of the left hemisphere, whereas they seem to engage similar space in the left parietal operculum. If an area is somatotopically organized and the BOLD signal from the hand stimulation and the foot stimulation is of comparable amplitudes, one should find a foot representation by examining the contrast of all foot versus all hand [(FD + FS $)-(\mathrm{HD}+\mathrm{HS})]$ in each subject. Conversely, one should see a representation of the hand by examining the contrast of all hand versus all foot $[(\mathrm{HD}+\mathrm{HS})-(\mathrm{FD}+\mathrm{FS})]$. The result was that areas $3 b$ and 1 were clearly somatotopically organized, whereas it was not obvious whether the remaining areas were as strongly somatotopical.

Another and more descriptive way to examine somatotopy is to evaluate the extent to which the cluster associated with the foot stimulation would overlap the cluster associated with hand stimulation. We re-examined the contrasts of all foot versus rest $[(\mathrm{FD}+\mathrm{FS})-2 R]$ and all hand versus rest $[(\mathrm{HD}+\mathrm{HS})-2 \mathrm{R}]$ in each subject. For each subject and each cytoarchitectural area, a ratio was calculated to show the extent of hand and foot cluster overlap. A high ratio indicates a large overlap of clusters and, therefore, little somatotopical organization. The ratio was calculated by the intersection of foot and hand activations divided by the union of these two activations (i.e., [(FD + FS) $2 R] \cap[(\mathrm{HD}+\mathrm{HS})-2 \mathrm{R}] /[(\mathrm{FD}+\mathrm{FS})-$ $2 R] \cup[(\mathrm{HD}+\mathrm{HS})-2 R])$. Only if both clusters within the respective cytoarchitec- 
Table 3. The effect of task-related attention

\begin{tabular}{|c|c|c|c|c|c|c|}
\hline \multirow[b]{2}{*}{ Contrast } & \multirow[b]{2}{*}{ Anatomical location of peak activity } & \multirow{2}{*}{$\begin{array}{l}\text { Cluster size }(\mathrm{mm} 3) \text { at } \\
\text { omnibus } p<0.05\end{array}$} & \multicolumn{3}{|c|}{$\begin{array}{l}\text { Talairach coordinate of the peak within the } \\
\text { cluster }\end{array}$} & \multirow[b]{2}{*}{ Voxel level Z-score } \\
\hline & & & $x$ & $y$ & $z$ & \\
\hline \multirow[t]{6}{*}{ HD versus HS } & $\mathrm{IP} 2,2$ & 283 & -50 & -38 & 32 & 5.30 \\
\hline & $\mathrm{IP} 1,2$ & 38 & 32 & -48 & 44 & 4.45 \\
\hline & IP 2 & 23 & 40 & -30 & 32 & 4.36 \\
\hline & 44 & 75 & -46 & -4 & 18 & 4.15 \\
\hline & 44 & 23 & 48 & 2 & 32 & 4.14 \\
\hline & $4 a, 4 p, 6$ & 21 & -46 & -8 & 32 & 4.12 \\
\hline \multirow[t]{2}{*}{ FD versus FS } & OP4 & 95 & 56 & -2 & 14 & 3.73 \\
\hline & OP4 & 70 & -52 & -12 & 16 & 3.57 \\
\hline
\end{tabular}

The contrasts of discrimination versus no-discrimination during both the hand and foot tasks were made with a random effects analysis. For the two contrasts of discrimination using the hand and foot, the cytoarchitectural location significant cluster size, Talairach coordinate, and Z-score are given.

tural area exceeded $800 \mathrm{~mm}^{3}$ was this entity calculated (see Materials and Methods). The results are depicted in Figure 4. Areas having a low ratio and, hence, a small overlap of hand and foot clusters indicating a high degree of separation of these regions were areas $3 a, 3 b, 1$, and 2 in decreasing order of somatotopy. The clusters encroaching into areas OP2 and OP3 were, in all subjects, so small for both hand and foot stimulations that they failed to reach the criterion. Therefore, it is not possible to make any statements about the somatotopy of these areas. For the hemisphere contralateral to the stimulated extremities, the ratio increased in the following order: IP1, OP4, IP2, OP1, indicating less and less somatotopy.

The use of a search space restricted to the somatosensory and motor areas of the brain does not permit one to examine whether there were representations of hand and foot outside these areas. When the search space was increased to cover the

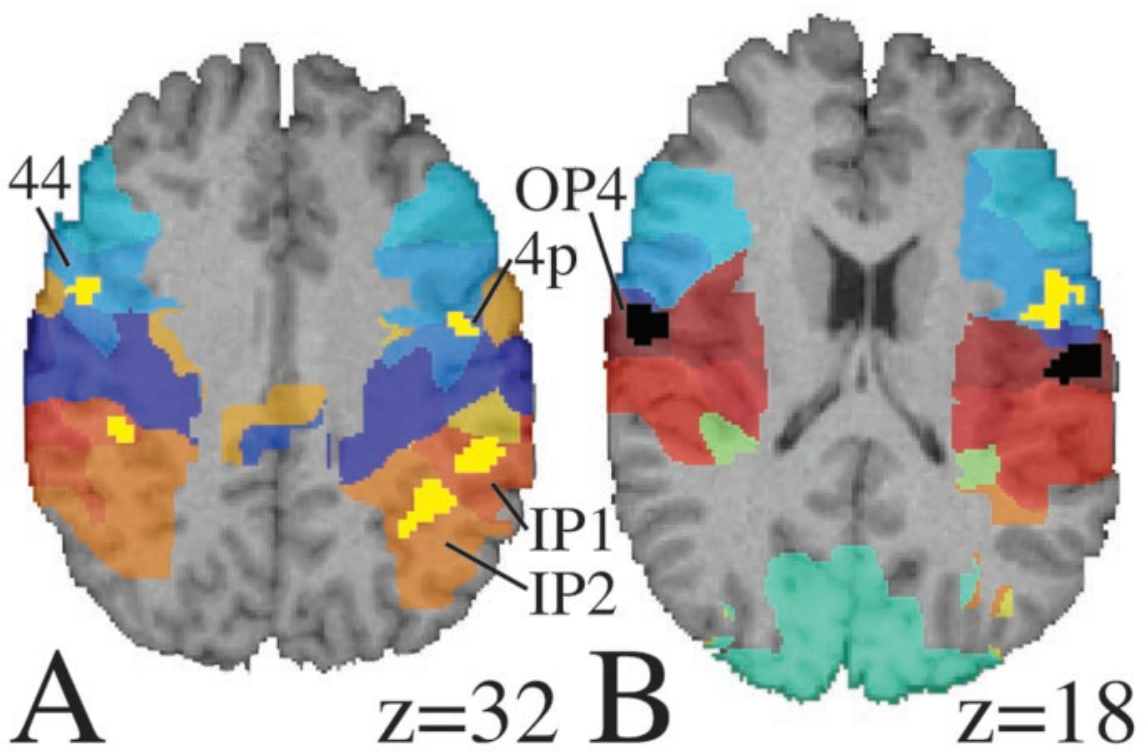

Figure 5. The discrimination component of hand and foot tasks. The contrasts of HD-HS (yellow) and FD-FS (black) are illustrated. These contrasts are overlaid on the cytoarchitectural probability map (translucent color coded), which is overlaid on the standard brain (gray scale) in Talairach space. whole space of the brain, and the significance limit was set to $p<0.05$ omnibus, there were representations of the hand and foot caudally to OP2, perhaps located to the retroinsular cortex (Roland and Mortensen, 1987).

\section{Group analysis: the effect of task-related attention}

Because no discrimination was required during the HS and FS conditions as identical objects were presented to either the right hand or foot, and because discrimination was required during the HD and FD conditions to discriminate between objects, one could isolate the discrimination component for the hand and foot tasks by contrasting these conditions. This analysis was performed as a random effects analysis of all subjects. The contrasts (HD - HS) and (FD - FS) were made. Significant clusters are listed in Table 3. For the (HD - HS) contrast, significant clusters were found in area 2, IP1, and IP2 (Fig. 5a). A contrast of (FD FS) revealed peaks in OP4 bilaterally (Fig. $5 b$ ).

\section{Discussion}

In this study, we examined the known somatosensory areas in the postcentral gyrus, 3a, 3b, 1 (Geyer et al., 1999), and 2 (Grefkes et al., 2001), parcellated by an observer-independent method (Schleicher et al., 1999). Somatosensory stimulation was performed on the immobile palm and planta. In addition, new and putative somatosensory areas, IP1, IP2, and OP1-OP4, parcellated cytoarchitecturally were examined for activations caused by stimulation of the hand and foot. All areas parcellated in the hemisphere contralateral to the stimulation did have representations of the hand and foot in the majority of the subjects, with the exception of areas 3a, OP2, and OP3. The right OP3 primarily contained a representation of the foot. In areas $3 \mathrm{~b}, 1$, and 2 , representations of the hand and foot were relatively distinct. Although the area 2 foot representation was on the superolateral side of the brain. Areas IP1, IP2, OP1, and OP4 had little or no somatotopical organization (Fig. 4a,b). Discrimination by the use of the foot as opposed to shape stimulation with identical objects enhanced the signal in OP4 bilaterally, whereas discrimination by the use of the hand enhanced the signal bilaterally in area 2, IP1, and IP2 (Fig. 5, Table 3).

A voxel in the probability map of the cerebral cortex only indicates the cytoarchitectural area found in the postmortem brains as being more frequently represented than any other area. For example, the space allocated to area IP1 in the probability map will also contain, with lesser but not negligible probability, representations of adjacent areas 2 and IP2. Furthermore, the individual brain images in this study were transformed into a standard anatomical space the same way, but not with the same software, as the postmortem brains. This might have caused a 
Ratio of hand/foot cluster overlap

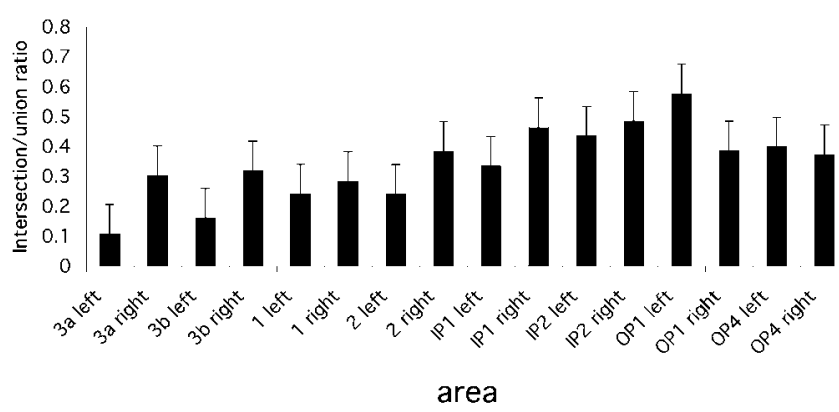

Figure 6. A ratio representing the common area shared by hand and foot representations for each cytoarchitecturally delineated area. The amount of overlap of the Boolean operations [(FD $+\mathrm{FS}-2 \mathrm{R}) \cap(\mathrm{HD}+\mathrm{HS}-2 \mathrm{R})] /[(\mathrm{FD}+\mathrm{FS}-2 \mathrm{R}) \cup(\mathrm{HD}+\mathrm{HS}-2 \mathrm{R})]$ on the $6 \mathrm{~mm}$ filtered cytoarchitectural probability map for each area for each subject was calculated. Ratios are calculated for areas 6, 4a, 4p, 3a, 3b, 1, 2, OP, and IP in both hemispheres in each subject. An average of the ratio across 10 subjects is shown. $A$ higher overlap between hand and foot activations in each area is reflected as a higher ratio.

slight discrepancy in the coregistration of the cytoarchitectural areas and the functional images. However, coregistration errors were small, for example, the positioning of the bottom of the central sulcus where the border between area $3 \mathrm{a}$ and $4 \mathrm{p}$ is located (Bodegard et al., 2001; Crivello et al., 2002). Smoothing the functional maps and cytoarchitectural maps compensated for any coregistration effects but at the expense of contaminating the borders of BOLD signals and cytoarchitectural areas in adjacent locations. Age-related brain modifications may also exist between the population of subjects in this experiment and the postmortem brain donors. Therefore, the cytoarchitectural areas of the probability map are no more than the best estimate of the location of these areas currently available.

Areas $3 \mathrm{a}, 3 \mathrm{~b}, 1$, and 2 in humans are regarded as somatosensory areas. This is because of their consistent responses to mechanoreceptive stimulation and previous evidence that these areas contain a full representation of the skin surface (Roland and Larsen, 1976; Greenberg et al., 1979; Reivich et al., 1983; Fox et al., 1987; Ginsberg et al., 1987). The present demonstration that each of the areas $3 \mathrm{~b}, 1$, and 2 contain a distinct representation of both the hand and the foot is new. This indicates that these areas in humans may be organized after the same scheme of multiple representations of the skin surface as in other primates (Kaas, 1983). However, we cannot conclude that areas 3a, 3b, 1, and 2 and areas IP1, IP2, OP1, and OP4 have a full representation of the skin surface, somatotopical or not, although they all have hand and foot representations and thus qualify as candidates for somatosensory areas. Areas IP1 and IP2 have been activated previously by tasks in which somatosensory stimulation has been predominant, but in these cases, the stimulation was presented to the hand (Roland et al., 1998; Bodegard et al., 2001). Here, the new finding is that these areas are also activated by somatosensory stimulation of the foot. The terminology has also been somewhat confusing as different labels, such as intraparietal area and anterior intraparietal area, and descriptive terms of localization within the supramarginal gyrus have been used for the anterior part of the IP region (Roland et al., 1998; Bodegard et al., 2000, 2001; Fink et al., 2000; Grefkes et al., 2002). Here, we propose that the terminology should be IP1 for the rostral area and IP2 for the area immediately caudal to IP1.

For area $3 \mathrm{a}$, less than half of the subjects had a representation of either the hand or the foot. This could be because our stimuli did not efficiently activate this area, which might be more readily activated by proprioceptive afferents (Naito et al., 1999, 2000, 2002). Thus, we cannot exclude that the failure to activate OP2 could have a similar cause, because temperature or roughness stimuli may be more appropriate for this region (O'Sullivan et al., 1994; Ledberg et al., 1995; Roland et al., 1998). In any case, our failure to activate these areas consistently does not imply that they are not somatosensory.

There have been no studies examining the somatotopy of the region just posterior to area 2. In the newly delimited areas IP1 and IP2, the hand and the foot representations overlapped, and the peak activities for hand and foot showed no consistent segregation. Each of these areas contains subdivisions that may be somatotopically organized but beyond the resolution of the fMRI method used, or that IP1 and IP2 are indeed single areas with large receptive fields and a coarse somatotopy or no somatotopy.

There are additional somatosensory areas in the parietal operculum and perhaps the retroinsular cortex, but their number and location in humans are still unresolved (Penfield and Rasmussen, 1950; Hari et al., 1983; Roland and Mortensen, 1987; Seitz and Roland, 1992; Burton et al., 1993, 1997; Lin et al., 1996; Hodge et al., 1998; Maldjian et al., 1999; Polonara et al., 1999; Disbrow et al., 2000; Francis et al., 2000). Investigation of secondary and associational somatosensory areas has been hindered by the selective nature of these areas and their tendency to respond only to certain stimuli. Activation of the parietal operculum (PO) has been reported by somatosensory discrimination of hardness (Servos et al., 2001), texture (Roland et al., 1998), electrical stimulation of finger and toes (Ruben et al., 2001; Del Gratta et al., 2002; Hagen and Pardo, 2002), and stimulation with moving sponges (Disbrow et al., 2001). At present, it is impossible to ascertain which of the areas OP1-OP4 would represent SII, the second somatosensory area, which at least in monkeys is somatotopically organized (Woolsey and Fairman, 1946; Whitsel et al., 1969; Friedman et al., 1980; Robinson and Burton, 1980; Burton et al., 1995; Krubitzer et al., 1995). A somatotopic organization of SII has been reported in humans (Disbrow et al., 2000; Ruben et al., 2001; but see Gelnar et al., 1998; Hagen and Pardo, 2002). However, cytoarchitectural delineation of human PO reveals four regions within the $\mathrm{PO}$, whereas these studies reported only a single somatotopical field.

From Figure 4, it is evident that motor areas PM, SMA, 4a, and $4 \mathrm{p}$ and area 44 were also activated. These motor activations may be attributable to the verbal response required from the subjects on completion of each condition; however, activation of motor areas during passive somatosensory stimulation has been reported previously (Naito et al., 1999; Bodegard et al., 2000; Oishi et al., 2003).

The present data do not support a clear somatotopical organization of the OP1 and OP4 for which we found strong responses from the hand and foot stimulation. Neither did the distribution of hand and foot peak activities in the parietal operculum give reasons to suggest another parcellation that could accommodate somatotopy. OP3 was mostly activated by stimulation of the foot ipsilaterally. Ledberg et al. (1995) found three separate representations of the hand in response to roughness discrimination and brief touch. The present data could be in agreement with this previous finding that was unable to demonstrate any somatotopy. We cannot be certain that this area simply did not respond to our stimulation of the hand and foot and is, in fact, a candidate for SII.

In summary, the present data suggest varying degrees of somatotopy. The decreasing segregation of the hand and foot peak 
activations (Fig. 6), increasing overlap of hand- and footassociated activation clusters (Fig. 6), and increasing merging of the hand and foot activations (Fig. 4a,b; Table 2) all suggest the same ranking of somatotopy: area $3 \mathrm{~b}$, area 1 , area 2 , area IP1, area OP4, area IP2, and area OP1. If the somatosensory cortex is organized according to the same principle as the visual cortex, areas that are low in the hierarchy will have strong topological organization, whereas areas high in the hierarchy will have little or no topological organization (Boussaoud et al., 1991). According to this, the above ranking of areas should reflect a somatotopical hierarchy of somatosensory areas. The fact that areas high in the hierarchy are more susceptible to attentional modulation than areas lower in the hierarchy is known from the visual cortex (Maunsell and Cook, 2002) and has also been observed in the somatosensory system (Fujiwara et al., 2002). Our finding of task-related attentional modulation of areas 2, IP1, and IP2 and of area OP4 is in accordance with a proposed somatotopical hierarchy.

\section{References}

Bodegard A, Geyer S, Naito E, Zilles K, Roland PE (2000) Somatosensory areas in man activated by moving stimuli: cytoarchitectonic mapping and PET. NeuroReport 11:187-191.

Bodegard A, Geyer S, Grefkes C, Zilles K, Roland PE (2001) Hierarchical processing of tactile shape in the human brain. Neuron 31:317-328.

Boussaoud D, Desimone R, Ungerleider LG (1991) Visual topography of area TEO in the macaque. J Comp Neurol 306:554-575.

Burton DB, Chelune GJ, Naugle RI, Bleasel A (1996) Neurocognitive studies in patients with supplementary sensorimotor area lesions. Adv Neurol 70:249-261.

Burton H, Videen TO, Raichle ME (1993) Tactile-vibration-activated foci in insular and parietal-opercular cortex studied with positron emission tomography: mapping the second somatosensory area in humans. Somatosens Mot Res 10:297-308.

Burton H, Fabri M, Alloway K (1995) Cortical areas within the lateral sulcus connected to cutaneous representations in areas $3 \mathrm{~b}$ and 1 : a revised interpretation of the second somatosensory area in macaque monkeys. J Comp Neurol 355:539-562.

Burton H, MacLeod AM, Videen TO, Raichle ME (1997) Multiple foci in parietal and frontal cortex activated by rubbing embossed grating patterns across fingerpads: a positron emission tomography study in humans. Cereb Cortex 7:3-17.

Choi H-J, Amunts K, Mohlberg H, Fink GR, Schleicher A, Zilles K (2002) Cytoarchitectonic mapping of the anterior ventral bank of the intraparietal sulcus in humans. NeuroImage 16[Suppl 1]:20038.

Corbetta M, Burton H, Sinclair RJ, Conturo TE, Akbudak E, McDonald JW (2002) Functional reorganization and stability of somatosensory-motor cortical topography in a tetraplegic subject with late recovery. Proc Natl Acad Sci USA 99:17066-17071.

Corkin S, Milner B, Rasmussen T (1970) Somatosensory thresholdscontrasting effects of postcentral-gyrus and posterior parietal-lobe excisions. Arch Neurol 23:41-58.

Crivello F, Schormann T, Tzourio-Mazoyer N, Roland PE, Zilles K, Mazoyer BM (2002) Comparison of spatial normalization procedures and their impact on functional maps. Hum Brain Mapp 16:228-250.

Del Gratta C, Della Penna S, Ferretti A, Franciotti R, Pizzella V, Tartaro A, Torquati K, Bonomo L, Romani GL, Rossini PM (2002) Topographic organization of the human primary and secondary somatosensory cortices: comparison of fMRI and MEG findings. NeuroImage 17:1373-1383.

Disbrow E, Roberts T, Krubitzer L (2000) Somatotopic organization of cortical fields in the lateral sulcus of Homo sapiens: evidence for SII and PV. J Comp Neurol 418:1-21.

Disbrow E, Roberts T, Poeppel D, Krubitzer L (2001) Evidence for interhemispheric processing of inputs from the hands in human S2 and PV. J Neurophysiol 85:2236-2244.

Eickhoff S, Geyer S, Amunts K, Mohlberg H, Zilles K (2002) Cytoarchitectonic analysis and stereotaxic map of the human secondary somatosensory cortex region. NeuroImage 16[Suppl 1]:20278.

Fink GR, Marshall JC, Shah NJ, Weiss PH, Halligan PW, Grosse-Ruyken M, Ziemons K, Zilles K, Freund HJ (2000) Line bisection judgments impli- cate right parietal cortex and cerebellum as assessed by fMRI. Neurology 54:1324-1331.

Foerster O (1936) Motorische felder und bahnen. In: Handbuch der Neurologie (Bumke O, Foerster O, eds), pp 50-51. Berlin: Springer

Fox PT, Burton H, Raichle ME (1987) Mapping human somatosensory cortex with positron emission tomography. J Neurosurg 67:34-43.

Francis ST, Kelly EF, Bowtell R, Dunseath WJ, Folger SE, McGlone F (2000) fMRI of the responses to vibratory stimulation of digit tips. NeuroImage 11:188-202.

Friedman DP, Jones EG, Burton H (1980) Representation pattern in the second somatic sensory area of the monkey cerebral cortex. J Comp Neurol 192:21-41.

Friston KJ, Holmes AP, Price CJ, Buchel C, Worsley KJ (1999) Multisubject fMRI studies and conjunction analyses. NeuroImage 10:385-396.

Fujiwara N, Imai M, Nagamine T, Mima T, Oga T, Takeshita K, Toma K, Shibasaki H (2002) Second somatosensory area (SII) plays a significant role in selective somatosensory attention. Brain Res Cogn Brain Res 14:389-397.

Gelnar PA, Krauss BR, Szeverenyi NM, Apkarian AV (1998) Fingertip representation in the human somatosensory cortex: an fMRI study. NeuroImage 7:261-283.

Geyer S, Ledberg A, Schleicher A, Kinomura S, Schormann T, Burgel U, Klingberg T, Larsson J, Zilles K, Roland PE (1996) Two different areas within the primary motor cortex of man. Nature 382:805-807.

Geyer S, Schleicher A, Zilles K (1999) Areas 3a, 3b, and 1 of human primary somatosensory cortex. NeuroImage 10:63-83.

Ginsberg MD, Yoshii F, Vibulsresth S, Chang JY, Duara R, Barker WW, Boothe TE (1987) Human task-specific somatosensory activation. Neurology 37:1301-1308.

Greenberg RP, Miller JD, Becker DP (1979) Early prognosis after severe human head injury utilizing multimodality evoked potentials. Acta Neurochir Suppl (Wien) 28:50-51

Grefkes C, Geyer S, Schormann T, Roland P, Zilles K (2001) Human somatosensory area 2: observer-independent cytoarchitectonic mapping, interindividual variability, and population map. NeuroImage 14:617-631.

Grefkes C, Weiss PH, Zilles K, Fink GR (2002) Crossmodal processing of object features in human anterior intraparietal cortex: an fMRI study implies equivalencies between humans and monkeys. Neuron 35:173-184.

Hagen MC, Pardo JV (2002) PET studies of somatosensory processing of light touch. Behav Brain Res 135:133-140.

Hari R, Hamalainen M, Kaukoranta E, Reinikainen K, Teszner D (1983) Neuromagnetic responses from the second somatosensory cortex in man. Acta Neurol Scand 68:207-212.

Head H, Holmes G (1911) Sensory disturbances from cerebral lesions. Brain 34:102-254.

Hodge Jr CJ, Huckins SC, Szeverenyi NM, Fonte MM, Dubroff JG, Davuluri K (1998) Patterns of lateral sensory cortical activation determined using functional magnetic resonance imaging. J Neurosurg 89:769-779.

Kaas JH (1983) What, if anything, is SI? Organization of first somatosensory area of cortex. Physiol Rev 63:206-231.

Kaas JH, Nelson RJ, Sur M, Lin CS, Merzenich MM (1979) Multiple representations of the body within the primary somatosensory cortex of primates. Science 204:521-523.

Krubitzer L, Clarey J, Tweedale R, Elston G, Calford M (1995) A redefinition of somatosensory areas in the lateral sulcus of macaque monkeys. J Neurosci 15:3821-3839.

Ledberg A, O'Sullivan BT, Kinomura S, Roland PE (1995) Somatosensory activations of the parietal operculum of man. A PET study. Eur J Neurosci 7:1934-1941.

Lin W, Kuppusamy K, Haacke EM, Burton H (1996) Functional MRI in human somatosensory cortex activated by touching textured surfaces. J Magn Reson Imaging 6:565-572.

Maldjian JA, Gottschalk A, Patel RS, Pincus D, Detre JA, Alsop DC (1999) Mapping of secondary somatosensory cortex activation induced by vibrational stimulation: an fMRI study. Brain Res 824:291-295.

Matsumura M, Kawashima R, Naito E, Satoh K, Takahashi T, Yanagisawa T, Fukuda H (1996) Changes in rCBF during grasping in humans examined by PET. NeuroReport 7:749-752.

Maunsell JH, Cook EP (2002) The role of attention in visual processing. Philos Trans R Soc Lond B Biol Sci 357:1063-1072. 
Naito E, Ehrsson HH, Geyer S, Zilles K, Roland PE (1999) Illusory arm movements activate cortical motor areas: a positron emission tomography study. J Neurosci 19:6134-6144.

Naito E, Kinomura S, Geyer S, Kawashima R, Roland PE, Zilles K (2000) Fast reaction to different sensory modalities activates common fields in the motor areas, but the anterior cingulate cortex is involved in the speed of reaction. J Neurophysiol 83:1701-1709.

Naito E, Roland PE, Ehrsson HH (2002) I feel my hand moving: a new role of the primary motor cortex in somatic perception of limb movement. Neuron 36:979-988.

Oishi K, Toma K, Matsuo K, Nakai T, Chihara K, Fukuyama H (2003) Cortical motor areas in plantar response: an event-related functional magnetic resonance imaging study in normal subjects. Neurosci Lett 345:17-20.

Oldfield RC (1971) The assessment and analysis of handedness: the Edinburgh inventory. Neuropsychologia 9:97-113.

O'Sullivan BT, Roland PE, Kawashima R (1994) A PET study of somatosensory discrimination in man. Microgeometry versus macrogeometry. Eur J Neurosci 6:137-148.

Penfield W, Boldrey E (1937) Somatic motor and sensory representation in the cerebral cortex of man as studied by electrical stimulation. Brain 60:389-443.

Penfield W, Rasmussen T (1950) The cerebral cortex of man: a clinical study of localization of function. New York: Macmillan.

Penfield W, Rasmussen T (1952) The cerebral cortex of man. New York: Macmillan.

Polonara G, Fabri M, Manzoni T, Salvolini U (1999) Localization of the first and second somatosensory areas in the human cerebral cortex with functional MR imaging. Am J Neuroradiol 20:199-205.

Reivich M, Gur R, Alavi A (1983) Positron emission tomographic studies of sensory stimuli, cognitive processes and anxiety. Hum Neurobiol 2:25-33.

Robinson CJ, Burton H (1980) Somatotopographic organization in the second somatosensory area of M. fascicularis. J Comp Neurol 192:43-67.

Roland E, Larsen B (1976) Focal increase of cerebral blood flow during stereognostic testing in man. Arch Neurol 33:551-558.

Roland P, Svensson G, Lindeberg T, Risch T, Baumann P, Dehmel A, Frederiksson J, Halldorson H, Forsberg L, Young J, Zilles K (2001) A database generator for human brain imaging. Trends Neurosci 24:562-564.
Roland PE (1975) Some principles and new methods of tactile stimulation. Behav Res Meth Instr 17:333-338.

Roland PE (1981) Somatotopical tuning of postcentral gyrus during focal attention in man. A regional cerebral blood flow study. J Neurophysiol 46:744-754.

Roland PE (1984) Organization of motor control by the normal human brain. Hum Neurobiol 2:205-216.

Roland PE, Mortensen E (1987) Somatosensory detection of microgeometry, macrogeometry and kinesthesia in man. Brain Res 434:1-42.

Roland PE, Zilles K (1998) Structural divisions and functional fields in the human cerebral cortex. Brain Res Brain Res Rev 26:87-105.

Roland PE, O'Sullivan B, Kawashima R (1998) Shape and roughness activate different somatosensory areas in the human brain. Proc Natl Acad Sci USA 95:3295-3300.

Ruben J, Schwiemann J, Deuchert M, Meyer R, Krause T, Curio G, Villringer K, Kurth R, Villringer A (2001) Somatotopic organization of human secondary somatosensory cortex. Cereb Cortex 11:463-473.

Schleicher A, Amunts K, Geyer S, Morosan P, Zilles K (1999) Observerindependent method for microstructural parcellation of cerebral cortex: a quantitative approach to cytoarchitectonics. NeuroImage 9:165-177.

Seitz RJ, Roland PE (1992) Vibratory stimulation increases and decreases the regional cerebral blood flow and oxidative metabolism: a positron emission tomography (PET) study. Acta Neurol Scand 86:60-67.

Seitz RJ, Roland PE, Bohm C, Greitz T, Stone-Elander S (1991) Somatosensory discrimination of shape: tactile exploration and cerebral activation. Eur J Neurosci 3:481-492.

Servos P, Lederman S, Wilson D, Gati J (2001) fMRI-derived cortical maps for haptic shape, texture, and hardness. Brain Res Cogn Brain Res 12:307-313.

Talairach J, Tournoux P (1988) Co-planar stereotaxic atlas of the human brain: an approach to cerebral imaging. Stuttgart: Thieme.

Wang G, Tanaka K, Tanifuji M (1996) Optical imaging of functional organization in the monkey inferotemporal cortex. Science 272:1665-1668.

Whitsel BL, Petrucelli LM, Werner G (1969) Symmetry and connectivity in the map of the body surface in somatosensory area II of primates. J Neurophysiol 32:170-183.

Woolsey CN, Fairman D (1946) Contralateral, ipsilateral and bilateral representation of cutaneous receptors in somatic areas I and II of the cerebral cortex of pig, sheep and other mammals. Surgery 19:684-702. 